\title{
The Fa(r)st or the Spurious - Pulmonary vein cycle length - Is it the fuel driving persistent AF, or simply just fumes?
}

\author{
Sandeep Prabhu ${ }^{1}$ and Louise Segan ${ }^{2}$ \\ ${ }^{1}$ Alfred Hospital \\ ${ }^{2}$ Baker Heart and Diabetes Institute
}

February 2, 2022

Title: The Fa(r)st or the Spurious - Pulmonary vein cycle length - Is it the fuel driving persistent AF, or simply just fumes?

Authors: Sandeep Prabhu ${ }^{1,2,3}$ and Louise Segan ${ }^{1,2,3}$

\section{Institutions:}

1. The Alfred Hospital, Melbourne, Australia

2. Baker Heart and Diabetes Institute, Melbourne, Australia

3. University of Melbourne, Melbourne, Australia

\section{Corresponding Author:}

A/Prof Sandeep Prabhu

Address: The Alfred Heart Centre

$3^{\text {rd }}$ Floor, Phillip Bock

The Alfred Hospital

55 Commercial Road, Melbourne

Australia, 3004

Phone: +61 390762000

Email: S.Prabhu@alfred.org.au

\section{Funding statement:}

A/Prof Sandeep Prabhu has received speaker fees from Abbott and has received reimbursement for a clinical advisory role from Biosense Webster. He receives research funding from the Australian National Health and Medical Research Council (NHMRC), the Australian National Heart Foundation, the Baker Heart and Diabetes Institute and the University of Melbourne. He has previously received fellowship funding from Abbott and Boston Scientific. Dr Louise Segan receives fellowship support from the Australian National Heart Foundation and the NHMRC.

\section{Disclosures}

No funding sources are relevant to this piece of work, and there are no conflicts of interest to disclose. 
Pulmonary vein (PV) isolation (PVI) remains the foundation of atrial fibrillation (AF) ablation, however, PVI durability remains lower in persistent compared with paroxysmal AF, irrespective of ablation strategy.[1] Indeed, despite demonstrable atrial electroanatomical remodeling, thought to be a major driver of AF maintenance, substrate modification techniques have not improved ablation outcomes beyond PVI-alone in persistent AF. $[2,3]$.

The question of the extent to which pulmonary vein electrical activity continues to contribute the maintenance of AF, rather than simply a trigger for its initiation, still remains largely unanswered. Alternatively, pulmonary vein electrical activity may simply be a 'passive' marker of atrial substrate, rather than an active determinant of AF outcomes. A significant part of the problem is the absence of a reliable, reproducible and feasible method to characterise pulmonary vein activity. It is in this context, that Spera and colleagues propose a novel approach to assessing pulmonary vein activity in AF.

Spera and colleagues propose a novel, non-automated methodology for AF CL evaluation, coined the FARS 10- $^{-}$ CL measurement, with the objectives of (1) evaluating the intra-observer reproducibility of this novel measurement approach; (2) comparison with traditional AF CL measurements; and (3) determining the predictive performance of the FARS 10 -CL measurement on long-term outcomes following wide antral PVI-only ablation in a prospective cohort of 100 patients with persistent AF. Measurements were conducted offline using manual method of measuring the mean of 10 consecutive "Fastest Atrial Repetitive Similar morphology signal CL" within a 1-minute observational window. Selection of the fastest 10 consecutive CLs was performed manually using visual assessment.

The study reported a high degree of intra-observer reproducibility utilising repeated $\mathrm{FARS}_{10}$-CL measurement across various left and right atrial structures and when compared with traditional AF CL measurement methodologies and a low reproducibility of traditional AF CL measurement of consecutive continuously fragmented CS signals.

Few studies have examined the relationship between AF cycle length (CL) and ablation outcomes with reported discrepant findings. Pascale et al performed a retrospective analysis of 97 patients with persistent AF and determined a PV to left atrial (LA) appendage (LAA) ratio $<69 \%$ predicted acute AF termination and was associated with more favourable ablation outcomes.[4] Conversely, Prabhu et al prospectively analysed 123 patients undergoing PVI with posterior wall isolation and found no clear association between PV activity and AF ablation outcomes in persistent AF.[5] Both studies involved non-automated PVCL measurement averaged over 100 consecutive beats.

These seemingly contrasting findings are likely driven by a paucity of data, relatively small population size and a distinct lack of a standardised approach to PVCL measurement. This is in part due to varying morphology and amplitude, significant CL fluctuations including sudden accelerations and organised activity, presence of fractionation obfuscating the dominant wavefront and poor inter-observer variability.

PV activity and AF recurrenceThe authors determined that a PV-FARS ${ }_{10}$-CL threshold of $140 \mathrm{~ms}$ (PVFARS $_{10}$-CL $>140 \mathrm{~ms}$ reflecting slower PV activity) in the fastest PV was associated with more marked atrial substrate (characterised by high burden low voltage zone (LVZ) during LA electroanatomical mapping) and was a good predictor for arrhythmia recurrence.

Perhaps not surprisingly, patients with faster PV activity $\left(\right.$ PV-FARS $1_{10}$-CL $<140 \mathrm{~ms}$ ) had more favourable clinical characteristics including younger age, lower CHADS2VASc score, small LA volume, less prevalent coronary artery disease, a greater likelihood of AF termination during PVI and a lower likelihood of arrhythmia recurrence. The authors suggested that regions of faster PV activity (PV-FARS $10^{-} \mathrm{CL}<140 \mathrm{~ms}$ ) may reflect host drivers of persistent $\mathrm{AF}$, which may help to identify individuals in whom a PVI-only strategy may be favourable.

When analysed by LVZ extent, individuals with a higher burden of LVZ ( $>16 \%)$ had higher CHADS2VASc score, lower left ventricular (LV) ejection fraction (LVEF) LVEF and slower PV-FARS 10 -CL. Moreover, of those with high burden LVZ, none met the critical threshold of PV-FARS ${ }_{10}-\mathrm{CL}<140 \mathrm{~ms}$. 
This analysis confirms the findings from previous studies that slower PV activity is associated with higher burden atrial substrate. Moreover, they reported relatively normal FARS ${ }_{10}$-CL measurements in other atrial structures including the SVC, RAA and LAA, despite slower PV activity, suggesting a possible preferential impact of LVZ on PV activity. They posited that slower PV activity may reflect regional PV electrical and structural remodeling preceding the remaining LA regions or may indicate the absence of fast PV drivers in patients with high LVZ burden. Additionally, while those with slow PV activity were more likely to have high burden LVZ and lower mean LVEF, as reported in previous studies, [6] the relationship between slower PV-FARS $_{10}$-CL activity and high LVZ burden extended to those with mid-range and normal EF without heart failure (HF), highlighting the utility of PV activity as a reliable and reproducible surrogate of atrial remodeling, irrespective of $\mathrm{HF}$ status.

Interestingly, while a high burden of LVZ, reflective of more advanced atrial substrate, was associated with slower PV-FARS ${ }_{10}$ CL along with traditional markers of AF recurrence, namely higher LA volume and CHADS2VASc score and lower LVEF, slower activity (FARS ${ }_{10}$-CL $>140 \mathrm{~ms}$ ) was the only independent predictor of arrhythmia recurrence and faster PV activity was associated with greater arrhythmia-free survival ( $85 \%$ vs $59 \%$ at 24 months, $\mathrm{p}=0.0018$ ).

The finding of $\mathrm{FARS}_{10}$-CL $<140 \mathrm{~ms}$ as the sole predictor of freedom from AF recurrence, where traditional predictors appeared to be non-contributory, is worthy of note, yet may be attributable to the relatively small and heterogeneous study population and the relative underrepresentation of the $\mathrm{FARS}_{10}$-CL group $<$ $140 \mathrm{~ms}$, which comprised only $27 \%$ of overall study population. One might expect that high LVZ burden would independently predict AF recurrence given the strong correlation between high LVZ burden and slower PV activity and the fact that high LVZ burden was only observed among those with PV-FARS ${ }_{10}-\mathrm{CL}>140 \mathrm{~ms}$. This would support the well described relationship between atrial remodeling and AF recurrence following PVI.[7,8] Moreover, previous studies have demonstrated an association between limited LA low voltage and AF termination during PVI, suggesting the absence of atrial low voltage or atrial remodeling may identify a subset of patients in whom PVI alone may suffice. $[9,10]$

Indeed, previous studies have reported significant associations between clinical predictors (patient factors, AF duration and LA characteristics) and AF ablation outcomes,[11] and given the small sample size and low prevalence of high burden LVZ in this study, this apparent finding probably warrants further exploration in larger studies, powered to more rigorously evaluate this relationship.

While the 24-month study follow up certainly provided more robust longitudinal evaluation of long-term outcomes, the reported arrhythmia-free survival is considerably higher than one would expect for an exclusively persistent $\mathrm{AF}$ population, given the mounting evidence from observational and randomised studies of $\mathrm{AF}$ ablation and outcomes in persistent AF. [8,12] This may be in part due to advances in ablation technologies and techniques as outlined by the study authors, though more likely reflects the lack of continuous or remote ambulatory rhythm monitoring as has been pursued in other similar studies of this nature, which almost certainly underestimates the true event rate and would not capture subclinical AF recurrence episodes.

One of the major issues recognised by the study authors was the effect of uninterrupted antiarrhythmic drug (AAD) therapy on PV CL assessment; whereby continued AAD therapy could potentially accentuate PVCL slowing in a heterogeneous fashion among individuals or groups. This is particularly relevant, given the subsequent analysis of clinical outcomes relies on a critical PVCL threshold, which may be confounded by a differential effect of AAD therapy.

Furthermore, existing methods, predominantly utilising offline, non-automated analysis, propose varying approaches to PVCL measurement.[4,5] The challenge of manually annotating the dominant wavefront lies in the chaotic nature of fibrillatory activity, which itself limits reproducibility owing to both intra- and inter-observer variability. This could be overcome by automated cycle length analysis which might provide intra-procedural data which could be valuable in guiding real-time decision-making with respect to ablation strategy. Future studies could integrate existing methods to automated PVCL analysis and enhance the translational impact of PVCL evaluation into clinical management. 
Overall, the authors are to be commended for a novel and informative analysis that fills a significant knowledge gap and may enhance the mechanistic understanding of drivers in persistent AF. This is the first study to evaluate the performance of a novel methodology compared to previously described methods. Moreover, this builds on exists work exploring predictors of long-term durability of a PVI-alone strategy in persistent $\mathrm{AF}$ and may help to stratify which individuals with persistent AF patients may benefit from PVI-alone or additional ablation strategies.

This analysis strengthens the concept of PV slowing as a reflection of atrial remodeling, however what remains uncertain, especially given the current paucity of evidence, is the optimal method of PVCL analysis and whether PV activity confers prognostic information with regard to ablation outcomes. Further studies could reconcile the apparent discrepancies in existing literature, develop a standardised and reproducible approach to PVCL measurement and clarify the utility of PVCL measurement during ablation of persistent AF. This will help to determine the real question - whether if, in fact, pulmonary vein electrical activity is truely the 'fuel' behind the maintenance of AF in the persistent AF population, or if it simply represent the passive 'fumes' of the more advanced atrial substrate in the persistent AF phenotype.

\section{References}

1. Brooks AG, Stiles MK, Laborderie J, et al. Outcomes of long-standing persistent atrial fibrillation ablation: a systematic review. Heart rhythm. 2010;7(6):835-846.

2. Verma A, Jiang C-y, Betts TR, et al. Approaches to catheter ablation for persistent atrial fibrillation. New England Journal of Medicine. 2015;372(19):1812-1822.

3. Vogler J, Willems S, Sultan A, et al. Pulmonary vein isolation versus defragmentation: the CHASE-AF clinical trial. Journal of the American College of Cardiology. 2015;66(24):2743-2752.

4. Pascale P, Shah AJ, Roten L, et al. Pulmonary veins to left atrium cycle length gradient predicts procedural and clinical outcomes of persistent atrial fibrillation ablation. Circulation: Arrhythmia and Electrophysiology. 2014;7(3):473-482.

5. Prabhu S, Kalla M, Peck KY, et al. Pulmonary vein activity does not predict the outcome of catheter ablation for persistent atrial fibrillation: A long-term multicenter prospective study. Heart rhythm. 2018;15(7):980-986.

6. Prabhu S, Voskoboinik A, McLellan AJ, et al. Biatrial electrical and structural atrial changes in heart failure: electroanatomic mapping in persistent atrial fibrillation in humans. JACC: Clinical Electrophysiology. 2018;4(1):87-96.

7. Marrouche NF, Wilber D, Hindricks G, et al. Association of atrial tissue fibrosis identified by delayed enhancement MRI and atrial fibrillation catheter ablation: the DECAAF study. Jama.2014;311(5):498-506.

8. Voskoboinik A, Moskovitch JT, Harel N, Sanders P, Kistler PM, Kalman JM. Revisiting pulmonary vein isolation alone for persistent atrial fibrillation: a systematic review and meta-analysis. Heart Rhythm. 2017;14(5):661-667.

9. Drewitz I, Willems S, Salukhe TV, et al. Atrial fibrillation cycle length is a sole independent predictor of a substrate for consecutive arrhythmias in patients with persistent atrial fibrillation. Circulation: Arrhythmia and Electrophysiology. 2010;3(4):351-360.

10. Jadidi AS, Lehrmann H, Keyl C, et al. Ablation of persistent atrial fibrillation targeting low-voltage areas with selective activation characteristics. Circulation: Arrhythmia and Electrophysiology.2016;9(3):e002962.

11. Berruezo A, Tamborero D, Mont L, et al. Pre-procedural predictors of atrial fibrillation recurrence after circumferential pulmonary vein ablation. European heart journal. 2007;28(7):836-841.

12. Ganesan AN, Shipp NJ, Brooks AG, et al. Long-term outcomes of catheter ablation of atrial fibrillation: a systematic review and meta-analysis. Journal of the American Heart Association.2013;2(2):e004549. 